\title{
Contribución al desarrollo de la epistemología de las Ciencias Forestales
}

\author{
Contribution to the development of the epistemology of the forest sciences
}

\author{
Rodrigo Arce ${ }^{1, *}$
}

Recibido: 05 enero 2020 | Aceptado: 16 mayo 2020 | Publicado en línea: 01 agosto 2020 Citación: Arce, R. 2020. Contribución al desarrollo de la epistomología de las Ciencias Forestales. Revista Forestal del Perú 35(1): 4-20. DOI: http://dx.doi.org/10.21704/rfp.v35i1.1472

\begin{abstract}
Resumen
El presente artículo tiene el propósito de aportar a la construcción de una epistemología forestal que oriente el accionar de los involucrados a los bosques. Para el efecto se desarrolla una breve caracterización de la epistemología a partir del cual se derivan preguntas para la reflexión. De la revisión se concluye que la construcción del conocimiento es contextual y obedece a los desafíos de cada época, reconociendo las tendencias que inciden en su desarrollo. En esta perspectiva tenemos un proceso de complejización de la epistemología forestal, de visiones fuertemente madereras se está pasando a visiones socioecosistémicas, en la que los bosques son apreciados por sus múltiples valores incluyendo los culturales y espirituales. De miradas disciplinarias se está pasando a miradas interdisciplinarias y transdisciplinarias que recogen la complejidad socioecosistémica de los bosques. De la primacía del conocimiento científico se está pasando a la valoración de la interrelación de saberes, donde todos los conocimientos participan activamente y respetuosamente en el desarrollo de las ciencias forestales. De orientaciones de conocimiento centrado exclusivamente a lo racional, se está pasando a reconocer que la construcción del conocimiento es un proceso social que articula la integralidad de la persona siendo importantes las consideraciones emocionales, culturales y espirituales. De ahí la importancia de favorecer la conexión entre las ciencias forestales y la filosofía especialmente en sus vertientes ontológicas, epistemológicas y éticas. Aunque muchos de los problemas forestales se explican desde su entorno, aún no existen los suficientes conocimientos sobre la dinámica de los bosques tropicales para asegurar la sustentabilidad ecológica del manejo forestal, por lo que es necesario acelerar y profundizar este tipo de investigaciones en las perspectivas planteadas.
\end{abstract}

Palabras clave: Bosques, complejidad, epistemología forestal, filosofía forestal, socioecosistémica

\footnotetext{
${ }^{1}$ Maestría en Ecología y gestión ambiental, Escuela de Posgrado de la Universidad Ricardo Palma (URP), Av. Benavides 5440, Santiago de Surco, Lima, Perú.

* Autor de Correspondencia: rarcerojas@yahoo.es
} 


\begin{abstract}
This article aims to contribute to the construction of a forest epistemology that will guide the actions of those involved in forests. For this purpose, a brief characterization of the epistemology is developed from which questions are derived for reflection. The review concludes that the construction of knowledge is contextual and responds to the challenges of each epoch, recognizing the trends that affect its development. In this perspective, we have a process of complex forest epistemology, from strongly timbered visions to socio-ecosynthetic visions, in which forests are appreciated for their multiple values, including cultural and spiritual ones. We are moving from disciplinary views to interdisciplinary and transdisciplinary views that reflect the socio-ecosystemic complexity of forests. From the primacy of scientific knowledge we are moving towards the valuation of the interrelationship of knowledge, where all knowledge participates actively and respectfully in the development of forest sciences. From orientations of knowledge centred exclusively on the rational, we are moving towards the recognition that the construction of knowledge is a social process that articulates the integral nature of the person, with emotional, cultural and spiritual considerations being important. Hence the importance of fostering the connection between the forestry sciences and philosophy, especially in their ontological, epistemological and ethical aspects. Although many forest problems are explained from their environment, there is still not enough knowledge about the dynamics of tropical forests to ensure the ecological sustainability of forest management, so it is necessary to accelerate and deepen this type of research in the perspectives presented.
\end{abstract}

Key words: Forests, complexity, forest epistemology, forest philosophy, socio-ecosystem

\section{Introducción}

Heredera de la tradición positivista de la ciencia, las ciencias forestales se han construido bajo los paradigmas de la objetividad, de la causalidad, de la linealidad, el determinismo y la predictibilidad (García 2008). Así se ha producido una fuerte separación entre las ciencias forestales y la filosofía, en donde la vertiente productivista de las ciencias forestales presenta una débil vinculación con la ética. Por ello es entendible que hasta ahora el tema de la epistemología forestal haya sido poco abordada desde el propio sector forestal. El presente artículo tiene el propósito de aportar a la construcción de una epistemología forestal, reconociendo que es un cometido mayúsculo y que requerirá el concurso de profesionales y académicos forestales y otras disciplinas con visión socioecosistémica. Esta es una tarea pendiente con indudables repercusiones en la formación de los profesionales forestales y en la administración pública forestal.

Hasta ahora la construcción del conocimiento forestal tiene una impronta marcadamente disciplinaria con orientación a lo biofísico, y con una débil orientación hacia el desarrollo social, aunque recientemente se ha podido apreciar en el Perú experiencias puntuales donde el conocimiento forestal presenta un enfoque interdisciplinario. Ver el bosque desde diversas perspectivas no es algo nuevo, por cuanto era la práctica del naturalista alemán Alexander von Humboldt (Sabogal 2019: 70).

Asimismo, la influencia del enfoque positivista ha separado los temas académicos de lo cotidiano en la realidad forestal, de tal manera que solo se considera ciencia aquello que pasa por el método científico y se subestima otro tipo de conocimientos, saberes y experiencias. Bajo esta premisa jerárquica la educación forestal también tiene un enfoque de transferencia, desde los que saben hacia los que no saben.

Ahora bien, es importante reconocer que los bosques están sujetos a una serie de presiones del entorno. Su conservación o deterioro está influenciado por factores sociales, institucionales, legales, culturales, económicos y, sobre todo, por los modelos de desarrollo hegemóni- 
cos de los cuales resulta difícil abstraerse. Pero más allá de la multidimensionalidad de las presiones que recibe el bosque, tenemos que tener en cuenta la complejidad de los bosques tropicales, la cual provoca que los conocimientos sobre su dinámica natural sean insuficientes para tomar decisiones en el manejo de estos bosques que garanticen su sostenibilidad.

\section{Materiales y Métodos}

El presente artículo ha sido generado a partir de una revisión bibliográfica y de las experiencias y reflexiones del autor en el ejercicio profesional forestal. El artículo se estructura a partir de las siguientes preguntas guía:

- ¿Qué se entiende por epistemología?

- ¿Cuál es el objeto de estudio de las ciencias forestales?

- ¿Cuál es la finalidad del estudio de las ciencias forestales?

- ¿Bajo qué paradigmas se ha gestado el conocimiento de las ciencias forestales?

- ¿En qué medida estos paradigmas iniciales inciden en el desarrollo de las ciencias forestales?

- ¿Qué cambios de paradigmas se han producido y cómo inciden en el desarrollo de las ciencias forestales?

Con base al desarrollo de estas preguntas se orientan las discusiones con el propósito de plantear la relevancia del conocimiento de la epistemología de las ciencias forestales en el desarrollo forestal acorde con los retos que plantea una sociedad del conocimiento.

La revisión histórica alude principalmente a Europa y los Estados Unidos por su fuerte influencia en el desarrollo de las ciencias forestales en Latinoamérica y la discusión está correlacionada con la experiencia peruana, que es el ámbito a partir del cual se desarrolla el presente artículo. Para la revisión se ha considerado artículos científicos y libros en español. Será importante contribuir al desarrollo de la epistemología forestal con referencia a publicaciones disponibles en los idiomas alemán e inglés.

\section{Resultados}

\section{Alcances conceptuales de la epistemología}

Se puede señalar que la epistemología es la rama de la filosofía que estudia el origen y el proceso seguido de legitimación (validez) de los conocimientos científicos por parte de la comunidad científica (Jaramillo 2003).

La epistemología estudia y evalúa los problemas cognoscitivos de tipo científico, es decir, estudia, evalúa y critica el conjunto de problemas que presenta el proceso de producción de conocimiento científico (Martínez y Ríos 2006: 4). Actualmente la epistemología tiene una concepción extendida que abarca la teoría del conocimiento científico, la lógica de la ciencia, la semántica de la ciencia, la metodología de la ciencia, la ontología de la ciencia, la axiología de la ciencia, y la ética de la ciencia (Zamudio 2012: 32, 33). Se debe reconocer que "el conocimiento científico, es un producto social, y no tiene más definición que la que le otorga el contexto social en el cual se genera" (García 2006: 120).

\section{Breve historia de las ciencias forestales}

Es indudable que los bosques han acompañado a los seres humanos en todo su proceso histórico tanto para satisfacción de necesidades materiales como culturales y espirituales (Zapata y Peña-Chocarro 1998: 88). Cuando los seres humanos deciden hacer de los bosques un nuevo campo especializado de conocimiento se da origen a las ciencias forestales.

Las Ciencias Forestales, llamada también Dasonomía, refiere al "estudio de las técnicas para cultivar y manipular las masas forestales a través de la aplicación de principios biológicos y ecológicos, para la producción continua de bienes y servicios para la sociedad". La Silvicultura es una rama de la Dasonomía, aunque en la literatura también aparecen como sinónimos (Hawley y Smith 1972). Es interesante mencionar que en el caso peruano se ha producido un proceso de diversificación por lo que al interés inicial de las ciencias forestales, este se ha ampliado hacia consideraciones ambientales ( $\mathrm{Fa}-$ cultades de Ingeniería Forestal y Ambiental) y 
agroforestales (Facultades Agroforestales). En otros casos las ciencias forestales se inscriben en Facultades Agrarias o Facultades de Recursos Naturales.

El origen de la profesión forestal parece situarse tanto en los guardabosques ingleses (principios del siglo XII), así como en los maestros de agua y bosques en Francia (principios del siglo XIII) (Sanz 2003). La dasonomía se desarrolló como ciencia en Alemania a fines del siglo XVIII de manera totalmente independiente de las ciencias agrícolas o pecuarias, época en la que se crearon las primeras escuelas forestales en lo que hoy es Alemania, Rusia, Suecia y Francia (del Valle 1997). En 1786 se creó la Academia de Tharand, fundada y dirigida por Heinrich Cotta en 1786, cuyo objetivo era la explotación racional de bosques (López 2016). En 1821 se creó en Alemania la Escuela Forestal de Berlín (Hasel 1985, citado por Sanz 2003: 161). La ciencia forestal se dedicaba al tratamiento de los espacios forestales conforme a principios técnicos y a la lógica científica, y nace como respuesta a la expansión de la agricultura, que ocasionaba el detrimento de los bosques y su devastación por usos tradicionales (Hasel 1989, citado por Sanz 2003: 162). Hay que señalar además que los primeros científicos forestales alternaban sus labores académicas con funciones en la administración pública, por lo que estas concepciones se trasladaron a la administración forestal (Sanz 2003: 163).

Francia también jugó un papel importante al haber involucrado el manejo de las cuencas hidrográficas dentro de las ciencias forestales, mientras que los alemanes lograron aportar en economía y medición forestal. Desde su inicio, la ciencia forestal mantuvo estrechas relaciones con las ciencias biológicas, económicas y matemáticas (del Valle 1997: 103).

\section{Objeto de estudio de las ciencias forestales}

Haciendo una síntesis de los objetos de estudios de las ciencias forestales se pueden reconocer las siguientes grandes categorías: Los bosques naturales, las plantaciones forestales, la industria forestal y la conservación de ecosistemas forestales. En la historia forestal también aparece el manejo de cuencas hidrográficas.

También existe el concepto de patrimonio forestal y de fauna silvestre que delimita el campo de competencias ligadas al sector forestal. En el caso peruano el patrimonio forestal y de fauna silvestre de la Nación está constituido por lo siguiente: a. Los ecosistemas forestales y otros ecosistemas de vegetación silvestre, b. Los recursos forestales y de fauna silvestre mantenidos en su fuente, c. La diversidad biológica forestal y de fauna silvestre, incluyendo sus recursos genéticos asociados, d. Los bosques plantados en tierras del Estado, e. Los servicios de los ecosistemas forestales y otros ecosistemas de vegetación silvestre, f. Las tierras de capacidad de uso mayor forestal y tierras de capacidad de uso mayor para protección, con bosques o sin ellos, y g. Los paisajes de los ecosistemas forestales y otros ecosistemas de vegetación silvestre en tanto sean objeto de aprovechamiento económico (Ley Forestal y de Fauna Silvestre $N^{\circ} 29763$, Art. 4).

\section{Finalidad del estudio de las ciencias fores- tales}

Los objetivos de las ciencias forestales han ido cambiando con el tiempo. Como menciona Prieto (2008: 33) inicialmente se atendía necesidades de "madera, leña y caza; después, productivas, protectoras, sociales y ambientales; y hoy en día, la función administradora de los forestales se extiende a la totalidad del espacio natural".

Beresford-Peirse en el año 1962 señalaba que dos son las preocupaciones básicas de los forestales: 1) Cómo habrán de satisfacerse las crecientes necesidades de madera en todas sus formas, y 2) Cómo podrán desempeñar los árboles y los montes su función protectora de salvaguardia de los recursos básicos de suelos y aguas (Beresford-Peirse 1962: 19). La concepción maderera de la actividad forestal aún se mantiene fuerte, aunque cada vez se ha ido reconociendo los valores múltiples de los bosques. Como señala Prieto (2008: 33):

Prácticamente durante todo el siglo XX, el objetivo de la ordenación de montes ha sido funda- 
mentalmente la obtención de productos basados en los postulados clásicos de la persistencia (que la masa ocupe el suelo sin interrupciones no programadas de antemano), rentabilidad (que se obtenga una renta anual o periódica constante) y máximo rendimiento (las masas se aprovechan a su edad de madurez o turno óptimo).

Siebert (1993) da cuenta que "las malas experiencias con plantaciones coetáneas llevaron a que se privilegie un enfoque de bosques multietáneos, los cuales en su estructura y su composición se asemejan mucho a los bosques nativos del sitio, lo que favoreció el desarrollo de una silvicultura más cercana a lo natural". De esta manera, la mirada centrada únicamente en la producción de bienes comercializables (monetariamente valorizables), se amplió para considerar las funciones ecológicas (incluyendo las no monetariamente valorizables en el largo plazo) (Peter 2000: 9).

Shirley (1964) al discutir sobre la creación de centros de enseñanza forestal deja entrever que los objetivos de la profesión forestal son: la ordenación forestal con fines de producción industrial de la madera, la protección de cuencas hidrográficas y la producción de la vida silvestre.

\section{Paradigmas bajo las que se ha gestado el conocimiento de las ciencias forestales}

En 1713 se acuña en Alemania el concepto de rendimiento sostenido (Malleux 2013: 15). Ese año Hans Carl von Carlowitz (1645-1714) (citado por Gonzáles-Doncel y Gil 2014: 23) publicó el libro Sylvicultura aeconomica que hacía referencia a la técnica de criar, cultivar y cortar los árboles. La sostenibilidad además estaba más acorde "con ideas procedentes del romanticismo alemán que vinculaba el mantenimiento de estos espacios con las esencias nacionales y con la belleza de la naturaleza primigenia" (Sanz 2003: 162).

Las ciencias forestales nacieron bajo el principio de la sostenibilidad (Malleux 2013: 15). La sostenibilidad implicaba la planificación de los turnos de la intervención de los bosques con el fin de entregar la producción maderable sin menoscabo del volumen y de la calidad a lo largo del tiempo. Según Sanz (2003: 161) el término sostenibilidad ("durabilidad") estaba más relacionado con el suministro constante de inmensas cantidades de madera que con inquietudes medioambientales. $\mathrm{Al}$ respecto señala Prieto:

En la gestión de producción sostenida, los bosques fueron gestionados para producir madera de forma constante y periódica; para ello, los sistemas de aprovechamiento eran bien de tipo forestal (basado en conseguir el crecimiento medio máximo) o de tipo económico-financiero (se maximiza el valor actual neto o la tasa interna de rendimiento) (2008: 39).

El bosque era considerado más como superficie maderera a explotar o una de caza a arrendar que como un ecosistema (Sanz 2003: 163). El propio H. Cotta, considerado como el padre de la Dasonomía señalaba en 1832 que, "el fin de la explotación privada de bosques era conseguir los mayores ingresos en las superficies forestales" (Sanz 2003: 163). Desde un principio, "la joven ciencia forestal mostró ambiciones de consolidación y se unió estrechamente a las necesidades de los Estados liberales en formación y de intereses madereros en proceso de crecimiento" (Sanz 2003: 161).

Reconociendo el foco maderero de la actividad forestal, Guariguata y Evans (2010) proponen que se debería ampliar la mirada hacia los productos forestales no maderables.

Se puede reconocer entonces que las ciencias forestales nacieron como parte del paradigma reduccionista de separabilidad y manipulabilidad de la naturaleza mediante el cual «un bosque se reduce a madera comercial y la madera se reduce a celulosa para las industrias que fabrican pulpa de madera y papel» (Shiva 1991: 47). No obstante, hacia mediados del siglo XIX comenzaron a aparecer corrientes críticas que señalaban la necesidad de ampliar la mirada del bosque de acuerdo a los múltiples intereses de los actores y propiciando el reencuentro con la naturaleza, dando pie a lo que sería posteriormente el enfoque conservacionista" (Sanz 2003: 165).

Los Estados Unidos tienen un papel en el cambio de concepciones forestales. Así, destaca la participación de Aldo Leopold (1887-1948), 
académico de la Universidad de Wisconsin y un especialista en el manejo de recursos forestales, quién generó la propuesta de la ética de la tierra mediante la cual la tierra no debiera ser considerada como una propiedad sino como una comunidad a la cual pertenecemos todos y por tanto, la preocupación moral debería incluir a los ecosistemas y sus habitantes no humanos. Esta concepción tuvo profunda influencia en el movimiento conservacionista (Kwiatkowska 2012: 49-51). Asimismo, Gifford Pinchot (1865-1946) fue uno de los pioneros en la articulación bosques y ambiente al proponer una "explotación sustentable" con base en el conocimiento científico, aunque mantiene la disyunción entre personas y recursos naturales en el marco de una ética utilitarista (Klier et al. 2017: 68).

Es posible reconocer a través de la historia los diferentes paradigmas del manejo forestal. Así, Aguirre (2015) reconoce los siguientes paradigmas: Protección, Rendimiento sostenido de madera (1850), Rendimiento Sostenido de Uso Múltiple (1950-60), Ecosistemas Forestales Naturales (1970-80), Manejo Ecosistémico de Bosques (1990).

Por su parte Kimmins (1997) reconoce de manera general las siguientes etapas de la concepción del tratamiento de los bosques: i) Explotación (Pre-Dasonomía), ii) Regulación, (Dasonomía Administrativa), iii) Manejo Forestal (Dasonomía basada en principios ecológicos), y iv) Forestería Social (Dasonomía social), entendida esta última como el reconocimiento de los valores del bosque según múltiples involucrados y no solo de los madereros. Para el caso específico de los bosques europeos reconoce las siguientes etapas: i) Bosques $\mathrm{Na}$ turales Originales, ii) Explotación, iii) Métodos tradicionales, iv) Producción sostenida, v) Uso múltiple y vi) Regreso a lo natural.

Gudynas (2002) reconoce los siguientes grandes hitos: i) Explotación forestal eficiente con base científica de Glifford Pinchot, ii) Relación ética con la tierra basada en el amor y el respeto y iii) Uso múltiple y reencuentro con la naturaleza.

La historia de las ciencias forestales va paralela a la historia de las ciencias en general. Así se puede afirmar que en el origen las ciencias forestales ha estado marcado por el cientifismo, el antropocentrismo y el desarrollismo. El cientifismo se explica por su tradición positivista con predominancia del paradigma simplificante de la ciencia, su autosuficiencia y el divorcio con la filosofía (Rojo et al. 2013). Este aspecto prácticamente no ha cambiado más allá de algunos cursos aislados de filosofía o de ética. Una excepción, por lo menos a nivel de documentos de gestión, refiere la Facultad de Ingeniería Agroforestal y Acuícola de la Universidad Intercultural de la Amazonía que reconoce explícitamente la consideración de un paradigma ecocéntrico y valora el enfoque de interculturalidad (UNIA 2011).

Aragón (2011) hace un recuento de la historiografía forestal inglesa, francesa, alemana, estadounidense y española. No obstante, los estudios sobre historia forestal, especialmente en Latinoamérica aún son escasos.

Jordan et al. (1999: 12) señalan que durante la época prehispánica la relación entre los hombres y los bosques podría calificarse de armónica en tanto se enmarcaba en la Cosmovisión de la Pachamama. Los autores refieren que durante el virreinato empezó el desencuentro en tanto los colonialistas "mostraban interés por los bosques para dos propósitos principales: combustible y madera para construcción y apuntalamiento de las minas" (Jordan et al. 1999: 16).

En la Historia ambiental del Perú (Siglos XVIII y XIX) Díaz et al. (2016: 183) anotan que los bosques en la colonia:

...Fueron percibidos desde dos perspectivas hasta cierto punto opuestas: una en la que el bosque es fuente de materias primas necesarias para el desarrollo y otra que lo relaciona con lo "salvaje", de modo que, de alguna manera, compite con las actividades y requerimientos del mundo "civilizado".

Como se puede apreciar en gran medida esta visión se mantiene en la actualidad.

Jordan et al. (1999: 32-33) muestran la evolución del desarrollo forestal andino entre 1960 y 2000 y dan cuenta que la historia forestal no 
es sino reflejo del marco político, institucional, tecnológico y económico. Se puede entrever además que las políticas forestales nacionales están influenciadas por las políticas forestales internacionales.

\section{Incidencia de los paradigmas dominantes en el desarrollo de las ciencias forestales}

El paradigma de rendimiento sostenido dio pie al concepto de sostenibilidad, aspecto que ahora está estrechamente ligado al desarrollo (el desarrollo sostenible). Prueba de ello son lo que anteriormente fueron los Objetivos de Desarrollo del Milenio y actualmente los Objetivos de Desarrollo Sostenible. No obstante, hay que señalar que el concepto de desarrollo forestal sostenible todavía es incipiente y que no está exento de dificultades, controversias y ambigüedades (Rodríguez 2003: 297).

En la historia forestal se ha ido pasando de la explotación forestal (extracción sin reposición) al manejo forestal y al manejo forestal sostenible, incluyendo los esquemas de certificación forestal voluntaria o los indicadores y criterios para el buen manejo forestal implementados, con mayor o menor convicción por los Estados.

Se ha podido apreciar que las ciencias forestales tienen dos orientaciones básicas: una productivista inicialmente relativa a la madera, pero abriéndose cada vez más a los servicios ecosistémicos en general, y otra perspectiva conservacionista. No obstante, estos campos no debieran verse absolutamente excluyentes porque un buen manejo forestal está intrínsecamente asociado a consideraciones de conservación.

Inclusive, aun hablando desde una perspectiva tradicional del manejo forestal concentrada en especies comerciales valiosas se requiere una mirada ecosistémica, que dé cuenta de la diversidad, los diferentes niveles de organización, y los rasgos funcionales de toda la comunidad ecosistémica, tal como lo propone la ecología funcional (Salgado-Negret 2015).

Como se ha podido reconocer el desarrollo de las ciencias forestales, en la perspectiva productivista, está inscrita en el desarrollo de las ciencias en general por tanto también está dominada por el espíritu de la objetividad, predictibilidad, linealidad y determinismo, propios de una ciencia normal. Forma parte también de un enfoque ontológico disyuntivo mediante el cual hay una absoluta separación entre el ser humano y la naturaleza bajo una ética utilitarista, los bosques son valiosos en la medida en la que satisfacen los intereses y las necesidades humanas (antropocentrismo). Finalmente se inscriben bajo una lógica del desarrollismo, porque postulan que con el auxilio de la tecnociencia se va a lograr un desarrollo permanente donde el sector forestal sea un contribuyente importante al desarrollo nacional. En esta perspectiva, actualmente el manejo forestal se inscribe en la lógica de la ecoeficiencia y de la modernización ecológica sustentada en la confianza de los instrumentos de la economía convencional y en el optimismo tecnológico (Fuente et al. 2011, 109).

En la perspectiva conservacionista, que a veces ha sido considerada como aislada de la orientación productivista, hay algunas diferencias. Aunque en la práctica también están presentes el cientifismo, el antropocentrismo y el desarrollismo, una mejor comprensión de las interacciones ecológicas de los ecosistemas y una mayor sensibilidad a los valores de la vida, dieron pie al desarrollo de una ética forestal por la cual se entiende que los valores de los ecosistemas están más allá del interés humano. Esta sensibilidad ética por los bosques no se ha trasladado necesariamente al sector forestal productivista, tanto en los profesionales forestales como en la organización de la administración pública de los bosques. Incluso se antepone lo forestal a lo ambiental. Tal es así que las exigencias ambientales son consideradas como barreras técnicas al libre comercio de productos forestales (Moreno 2014: 75).

No obstante, la historia de la conservación tampoco está exenta de contradicciones y tensiones. En nombre del interés nacional se declaraban áreas protegidas sobre territorios ocupados por pueblos indígenas y por tanto se sustentaba su desplazamiento. Se caía por tanto en un culto a lo silvestre que desconocía a 
los pueblos indígenas en nombre de la conservación. Esta concepción está en retirada, aunque no totalmente pues todavía subsisten posiciones que señalan la conveniencia de áreas protegidas libres de pueblos indígenas.

Asimismo, la caza deportiva ha sido planteada como una medida positiva de conservación al basarse en la transferencia de mortalidad natural, en cosecha con generación de ingresos en beneficio de la propia conservación. Cohn (2012: 44) condena la caza basada en las siguientes razones:

...Conlleva un desperdicio completamente innecesario y gratuito de la vida sensible, altera el medioambiente de múltiples maneras, presenta e institucionaliza la violencia como un pasatiempo y modo de resolver problemas, y convierte la visión común del dominio sobre los animales en una dominación despiadada.

\section{Cambios de paradigmas y su incidencia en el desarrollo de las ciencias forestales}

En teoría el principio de la sustentabilidad rige el accionar de los profesionales forestales, no obstante, actualmente no existe suficiente certeza que se esté asegurando la sostenibilidad ecológica de los bosques tropicales con el aprovechamiento forestal, pese a los importantes avances en el desarrollo de las ciencias forestales, la tecnología, la legislación de promoción y sanciones a los delitos forestales, y los esquemas de certificación forestal voluntaria. Múltiples son los factores que explicarían un ejercicio de sustentabilidad débil (Dourojeanni 2015: 5), entre ellos se podría señalar la ontología disyuntiva que separa al ser humano de los bosques (que lo cosifica y legitima su explotación solo en el interés humano), la imposición de un esquema de desarrollo que privilegia la dimensión económica y subordina las consideraciones sociales y ambientales, los insuficientes conocimientos sobre la complejidad de los bosques tropicales, la administración forestal débil (Dourojeanni 2015: 5), la corrupción expandida (Dourojeanni 2015: 7-8), entre otros. La Figura 1 muestra el necesario cambio de visión en las relaciones entre los humanos y los bosques.

Merece especial atención el tema ético. Se aprecian casos de relajamiento ético producto de un paradigma de desarrollo individualista, materialista y pragmático. En otros casos se aprecia un divorcio entre gestión forestal y ética forestal, producto de una lógica económica

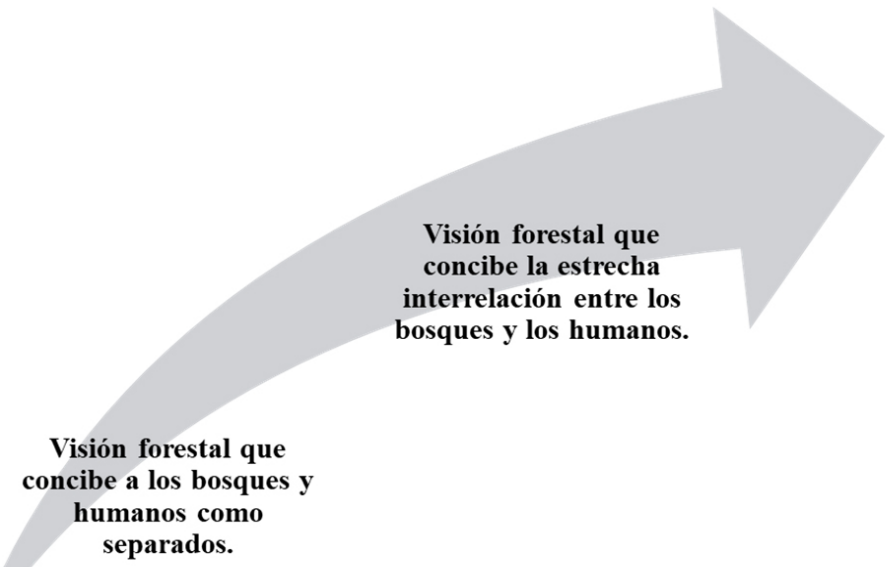

Figura 1. El cambio de las relaciones disyuntivas entre los bosques y los seres humanos a una relación de continuidad. 


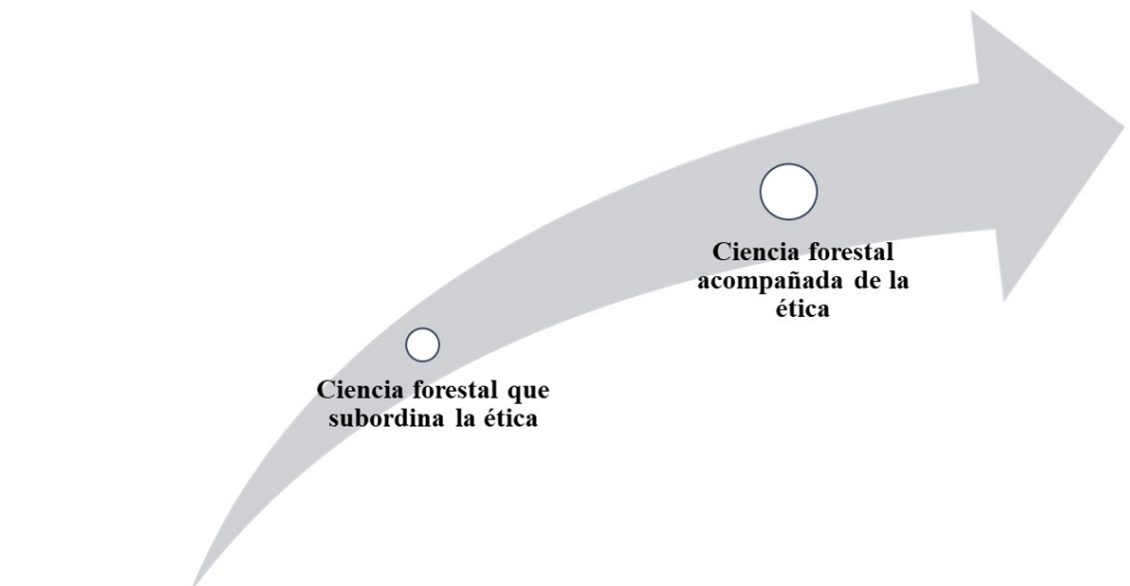

Figura 2. El cambio de una ciencia forestal alejada de la ética a una ciencia forestal acompañada de la ética.

predominante que debilita las consideraciones sociales y económicas. Para una perspectiva científica positivista, que aún predomina en las ciencias forestales, lo moral alude a valores subjetivos y por tanto le resta objetividad y por tanto de rigor científico. Asimismo, el positivismo rechaza cualquier atisbo de sensibilidad que corresponde al mundo emocional. La Figura 2 muestra el cambio de una ciencia forestal alejada de la ética a una ciencia forestal acompañada de la ética.

Aunque como se ha podido apreciar en la historia forestal, la conservación forma parte de las ciencias forestales que en la práctica se ha ido separando de tal manera que cuando se habla de lo forestal, se alude inmediatamente a la producción maderera, y ahora al carbono forestal, mientras que cuando se habla de conservación, se asocia inmediatamente a las áreas protegidas y se lo adscribe a posiciones ambientalistas. Incluso se llega a plantear al ambientalismo o al ecologismo como factores que perturban el desarrollo forestal. Las evidencias indican que para el mejoramiento de las actuales prácticas de manejo se requiere de una pragmática colaboración entre los ecologistas y los administradores forestales (Sheil y Meijaard 2005). Esteve (2015: 49) señala que:

...las razones que justifican la conservación de la naturaleza pueden ser resumidas en 4-E: las razones económicas (rendimientos), las ecológicas (servicios), las estéticas (paisaje) y las éticas (extinción de la biodiversidad). No obstante, todas ellas están atravesadas de componentes económicos y ecológicos y en todas ellas conviven en diferente proporción aspectos emocionales y racionales.

No solo se trata de promover un enfoque que reconozca las interacciones entre ciencias naturales y ciencias sociales sino también reconocer que nos encontramos en un mundo altamente complejo, interconectado, interdependiente y con grandes avances tecnológicos. El reto es cómo garantizar la conservación de los bosques en un planeta con un crecimiento exponencial de su población y por tanto, de necesidades objetivas y subjetivas a partir de los bosques. Por su parte, las comunidades rurales esperan, a la vez que se respeten plenamente sus derechos, que los bosques les permitan salir de la pobreza y la marginación (Villalobos et al. 2012).

\section{Discusión}

\section{Las falsas tensiones entre la especialización y la visión totalizadora}

La época actual está fuertemente dominada por el individualismo, el materialismo y el pragmatismo. La sociedad tiene preferencia por opciones prácticas, con resultados rápidos 
y rentables (Bauman 2004). Esta mirada está provocando que los indicadores de éxito se midan en términos económicos más que en el desarrollo de una vida plena a nivel personal y social. En este marco hay un fuerte desafecto por la filosofía y todo aquello que distraiga en el objetivo supremo de crecimiento económico. El ejercicio profesional forestal no está exento de estas presiones y tendencias.

Ampliar la mirada forestal desde la perspectiva de los bosques como socioecosistemas no implica subestimar la necesaria mirada especializada sino resignificarla y establecer estrategias para aprovechar lo mejor de cada perspectiva. De lo que se trata es entrar en un proceso de diálogo que permita la complementariedad y las sinergias entre los subsistemas ecológicos y sociales. Importa tanto la ciencia básica como la ciencia aplicada, la orientación científica como la orientación filosófica, la perspectiva técnica como la perspectiva política. La Figura 3 muestra los bosques concebidos como socioecosistemas.

El llamado desarrollo forestal no está exento de consideraciones geopolíticas, en tanto las tierras forestales se convierten en campo de disputa de intereses de diversos sectores que no es posible desconocer (Velasco y Velázquez
2019). Por tanto, es necesaria una mejor articulación no solo al interior de los sectores públicos sino también entre sectores y entre políticas públicas orientadas al desarrollo forestal y a la conservación de la biodiversidad (Zanetti et al. 2017).

\section{Sugerencias para una epistemología de las ciencias forestales acorde con los retos de es- tos tiempos}

Se plantea recuperar el sentido de la sostenibilidad que ha guiado el accionar de las ciencias forestales en los últimos 300 años y hacerlo más explícito y desarrollado en la formación de los profesionales forestales (Malleux 2013: 17).

Como señala Malleux (2013: 16) “el desafío es comprender que, ante la visión general de que la forestería es parte integral del manejo de los recursos naturales, el alcance de los campos de acción del sector forestal debe ser más amplio que el enfoque tradicional". Como menciona Dourojeanni $(2009,2015,2019)$ al hablar de bosques, usualmente se piensa en extracción de madera y formas derivadas, pero el reto es pasar a la generación de servicios ambientales sumamente importantes para el mantenimiento de los ecosistemas mundiales. Dourojeanni (2015: 17) menciona que "los bosques naturales

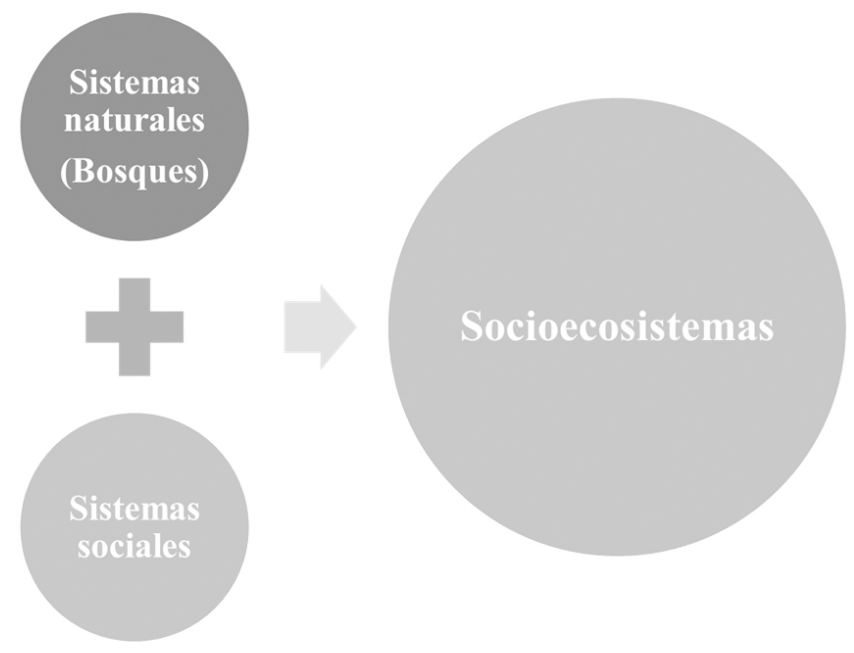

Figura 3. Los bosques como socioecosistemas. 
abiertos a la explotación maderera deberían ser sometidos a verdaderos planos de manejo y ser dedicados a producir volúmenes moderados de madera certificada de altísimo valor". La Secretaría de la Convención de las Naciones Unidas de Lucha contra la Desertificación UNCCD (2017: 45) reconoce que "las prácticas de manejo más intensivas en los bosques naturales o la conversión a plantaciones alteran la ecología y la hidrología y, si están mal planeadas, pueden conducir a la erosión del suelo y a la pérdida de otros servicios ecosistémicos".

Esto significa ampliar la mirada de cara a cada uno de los retos de los Objetivos de Desarrollo Sostenible de las Naciones Unidas más allá del objetivo 15 (Vida de Ecosistemas Terrestres). En el ámbito nacional significa no quedarse únicamente como un buen manejador de bosques, sino participar activamente en el desarrollo sostenible (Malleux 2014: 8). Sin embargo, es necesario considerar el agotamiento de las perspectivas de desarrollo o desarrollo sostenible inclusive puesto que, aunque hablen de consideraciones sociales y ambientales, mantienen la primacía de un enfoque economicista. De ahí que existan perspectivas postdesarrollistas o de alternativas al desarrollo que tratan de superar las visiones antropocéntricas y utilitaristas por una posición biocéntrica donde se ponga la vida como el centro del accionar humano, tomando en cuenta la vida de los humanos y la vida de los no humanos. En esta perspectiva se ubica las propuestas de ontologías relacionales, que dan cuenta de aquellas ontologías que revaloran las relaciones de continuidad entre la sociedad humana y la naturaleza. Actualmente, desde la perspectiva de la biología, se considera que la epigenética incorpora en una sola unidad lo que anteriormente estaba separado: la naturaleza y la cultura (Aristizábal et al. 2019: 55). La Figura 4 muestra la interrelación entre los bosques y el conjunto de Objetivos de Desarrollo Sostenible de Naciones Unidas.

En tanto se sigan sustentando las alternativas al desarrollo es posible usar como marco de referencia los Objetivos de Desarrollo Sostenible partiendo de una visión socioecosistémica de los bosques. Este enfoque permite hacer una conexión más directa con los otros objetivos que no se perciben producto de una visión sectorial y disciplinaria. Esto significa aceptar que los profesionales forestales no pueden asumir solos el desafío de manejar sosteniblemente los bosques y conservarlos (Dourojeanni 2015: 6). En esa dirección Hhilm, citado por Malleux (2014: 23), considera que el perfil del profesional forestal debería considerar la capacidad para "Interpretar y evaluar la realidad natural, social, económica y política del entorno donde se desempeña, incluyendo la percepción de las poblaciones locales para lograr la gobernanza del territorio".

\section{Bosques}

\section{Objetivos de Desarrollo Sostenible}

Figura 4. Interrelación entre los bosques y el conjunto de Objetivos de Desarrollo Sostenible de Naciones Unidas. 
El reconocimiento de la sintiencia en animales y la sensibilidad en las plantas es algo que no estaba considerado hasta ahora en la búsqueda de conocimiento de las ciencias forestales (Rincón 2018, Mancuso y Viola 2015). Desde la perspectiva de la disyunción entre el ser humano y los bosques, estos son tratados como recursos naturales sujetos a explotación al servicio del ser humano. Por lo tanto, no había que incluir consideraciones morales en la relación con los bosques. Con la ética de la tierra y la ética del cuidado extendida a la naturaleza existen nuevos elementos para el relacionamiento con los bosques (Pérez 2016). No en vano, cada vez se ha ido ampliando la visión forestal centrada exclusivamente a la madera a la consideración a los valores múltiples de los bosques, incluyendo valores culturales y espirituales que privilegian la desmaterialización del valor del bosque. De otro lado, desde las perspectivas de la prospectiva aplicada al sector forestal, indican que se requiere orientar la producción forestal a partir de los bosques naturales en pie, plantaciones forestales, o en todo caso, dar mayor valor agregado a la producción forestal incluyendo la base del conocimiento.

Para avanzar en las ciencias forestales se requieren tres retos fundamentales. 1) Reconocer que cuando se habla de sostenibilidad no es una palabra acabada que justifique la débil sustentabilidad ecológica del manejo de los bosques, y por ello se requiere una auténtica sustentabilidad fuerte en el sentido de una ecología profunda, 2) Reconocer la complejidad de los bosques y su relación estrecha con la sociedad, lo que sustenta la importancia de los enfoques socioecosistémicos (Rodríguez 2003), 3) Reconocer que la complejidad socioecosistémica de los bosques demanda conocimientos más profundos para lograr que la sustentabilidad se base en buena ciencia y para ello será necesario realizar procesos de investigación interdisciplinaria y transdisciplinaria (Aguirre-Calderón 2015: 26). Por ejemplo, no existen suficientes conocimientos de los bosques como sistemas disipativos alejados del equilibrio (Briones 2011, 264). Esto quiere decir que la dinámica de los ecosistemas forestales no está en equilibrio, sino en continuo cambio, sin un orden establecido (Botkin 1990; citado por Gudynas 2002: 29). La Figura 5 muestra la complejidad de los bosques como socioecosistemas.

Complementariamente será necesario una mayor fusión entre la ciencia forestal y la filosofía, relación que hasta ahora ha sido subestimada. Aunque la ciencia pretende objetividad absoluta no se puede sustraer de la forma cómo

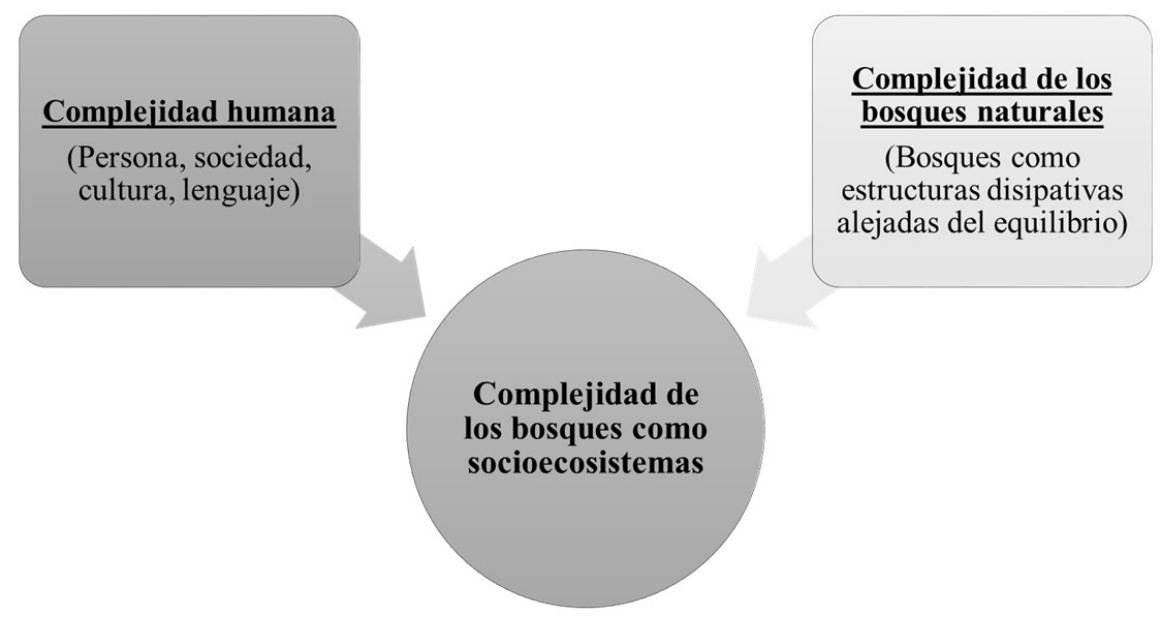

Figura 5. Complejidad de los bosques como socioecosistemas. 


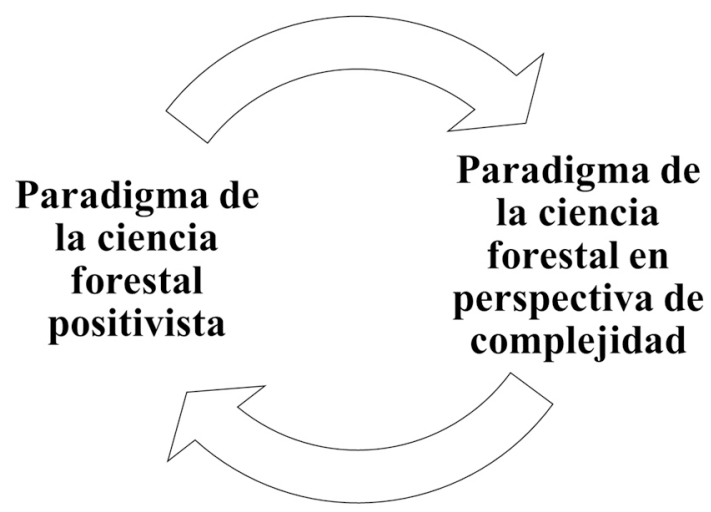

Figura 6. El necesario diálogo entre una perspectiva positivista y la perspectiva de complejidad de las ciencias forestales

percibimos, interrogamos y organizamos nuestra experiencia de la realidad. Captar la realidad desde el pensamiento científico no obvia el hecho que también se sustenta en cosmovisiones o filosofías implícitas (Rodríguez Zoya 2016: 43-44). Desde una perspectiva de ciencias de punta no hay división entre ciencias duras ni ciencias blandas y lo que se verifica más bien son fusiones colaborativas, integradoras y flujos de continuidades (Arce 2019: 36). Se impone por tanto un pensamiento de síntesis (Maldonado 2015: 29). De todo ello se desprende la necesidad de tomar más en cuenta la filosofía en el desarrollo de las ciencias forestales.

Aunque el paradigma científico de la simplicidad ha sido importante, y lo seguirá siendo, no es suficiente para dar cuenta de la complejidad de los socioecosistemas y por tanto se requiere una mayor articulación de las ciencias forestales a las ciencias de la complejidad y el pensamiento complejo. En esta perspectiva los enfoques de los sistemas complejos adaptativos resultan muy promisorios. La Figura 6 muestra el necesario diálogo entre una perspectiva positivista y la perspectiva de complejidad de las ciencias forestales.

No es posible terminar estas discusiones sin abordar los temas relativos al tratamiento del conocimiento forestal. De una visión que se- para el conocimiento occidental de los saberes y experiencias locales es necesario pasar a una interrelación de saberes a través de la cual la producción del conocimiento se realiza a través de las comunidades de pares en las que todos los actores tienen cosas importantes que decir (de Sousa Santos 2011). Esta perspectiva está más a tono con la realidad pluricultural de los países andinos demandantes de enfoques de interculturalidad. Esta propuesta también está más acorde con las corrientes de ciencia ciudadana (Piña 2017).

También es necesario reconocer que la construcción del conocimiento no alude únicamente a procesos estrictamente racionales, $y$ que el conocimiento involucra a toda la persona en su integralidad, siendo un proceso de construcción social. Por tanto, más allá de cuestiones racionales, también es necesario reconocer la importancia de los procesos de cognición de los sistemas socioecológicos (Jones et al. 2016).

Quiere decir entonces que es necesario reconocer que la construcción del conocimiento se da entre las universidades, la administración pública forestal y los usuarios forestales. En este círculo de continuidad el proceso de generación, producción y socialización de conocimientos se articula con el proceso de aplicación de conocimientos y no hay tal división entre los que enseñan y los que aprenden, pues son roles intercambiables. 
Desde la perspectiva del pensamiento ambiental latinoamericano se está recuperando la relación estrecha entre naturaleza y sociedad, se valoran los territorios y se valora la vida en todas sus manifestaciones. El reconocimiento de esta realidad exige nuevas maneras de pensar, para logar la ampliación de la conciencia (Cardona y Mora 2018: 45).

Marczak et al. (2016: 40) consideran que pese a las importantes medidas de conservación de bosques que se están adoptando en América Latina éstas no serán suficientes, por cuanto la presión de tierras agrícolas para producción de alimentos seguirá siendo importante, originando que la dotación de tierras forestales de la región disminuirá hacia el año 2030. Ello obliga a plantear políticas públicas que concilien ambos objetivos. Algunas de estas políticas, entre otras, tienen que ver con la adopción de enfoques territoriales; integración de políticas en materia de bosques, agricultura, alimentación, uso de la tierra y desarrollo rural y nacional; garantizar los derechos de tenencia de la tierra a las personas pobres y vulnerables, en particular los pueblos indígenas, los agricultores sin tierras y las mujeres y los jóvenes del medio rural (FAO 2018).

En este marco es posible entender por qué existe un correlato entre el desarrollo forestal con los enfoques de paisajes forestales sostenibles, el ordenamiento territorial y la gestión de cuencas.

Aunque actualmente existe una tendencia sostenida en el crecimiento del mercado de la madera hay que tener presente las tendencias que van en las siguientes direcciones: producción de alimentos a partir de celulosa, producción de biocombustibles a partir de la madera y el hecho de tener una mayor conciencia sobre la necesidad de mantener los bosques naturales en pie por sus múltiples valores. En una economía del conocimiento ya no es posible plantear el desarrollo forestal exclusivamente desde una mirada de proveedores de materias primas, que además están siendo reemplazados por la biología sintética. De ahí la pertinencia de vincular la epistemología forestal con estudios de prospectiva forestal.

\section{Conclusiones}

La construcción del conocimiento es contextual y obedece a los desafíos de cada época, reconociendo las tendencias que inciden en su desarrollo. En esta perspectiva tenemos un proceso de complejización de la epistemología forestal, de visiones fuertemente madereras se está pasando a visiones socioecosistémicas, en la que los bosques son apreciados por sus múltiples valores incluyendo los culturales y espirituales. De miradas disciplinarias se está pasando a miradas interdisciplinarias y transdisciplinarias que recogen la complejidad socioecosistémica de los bosques. De la primacía del conocimiento científico se está pasando a la valoración de la interrelación de saberes, donde todos los conocimientos participan activamente y respetuosamente en el desarrollo de las ciencias forestales. De orientaciones de conocimiento centrado exclusivamente a lo racional, se está pasando a reconocer que la construcción del conocimiento es un proceso social que articula la integralidad de la persona siendo importantes las consideraciones emocionales, culturales y espirituales. De ahí la importancia de favorecer la conexión entre las ciencias forestales y la filosofía especialmente en sus vertientes ontológicas, epistemológicas y éticas. Aunque muchos de los problemas forestales se explican desde su entorno, aún no existen los suficientes conocimientos sobre la dinámica de los bosques tropicales para asegurar la sustentabilidad ecológica del manejo forestal, por lo que es necesario acelerar y profundizar este tipo de investigaciones en las perspectivas planteadas.

\section{Bibliografía}

Aguirre, O. 2015. Manejo Forestal en el Siglo XXI. Madera y Bosques 21:17-28.

Aragón, Á. 2011. La historiografía forestal sobre época moderna en el panorama internacional, español y vasco: una revisión bibliográfica. Vasconia 37:117-140.

Arce, R. 2019. Consideraciones para el enriquecimiento del pensamiento complejo a partir de las críticas de Carlos Eduardo Maldo- 
nado Castañeda. Complessitá. Anno XIII (12):26-45. ISBN 978-88-7442-930-1.

Aristizábal, C; Bonilla, JV; Cárdenas, H; Galvis, S; Gómez, LA; Maldonado, CE; García, AC; Sandoval, J. 2019. La epigenética y la transformación radical de la biología. Bogotá, Colombia, Universidad El Bosque, Facultad de Medicina, Grupo de Investigación Complejidad y Salud Pública, 56 p.

Bauman, Z. 2004. Modernidad líquida. México, Fondo de Cultura Económica, 178 p.

Beresford-Peirse, H. 1962. La evolución de la dasonomía. Últimas tendencias en el concepto de dicha ciencia UNASYLVA 16 (67):19-23.

Briones, M. 2011. Ecosistema como estructura disipativa. In Conde, A; Ortiz, P; Delgado, A; Gómez, F; Granados, L. (Coordinadores). Primer Congreso Nacional Naturaleza y Sociedad. Memoria del Congreso. Tlaxcala, México. p. 256-270.

Cardona, CM; Mora, WM. 2018. Sostenibilidad/sustentabilidad una mirada diferenciadora desde el pensamiento ambiental latinoamericano. Bogotá, Colombia, Editorial Universidad Distrital Francisco José de Caldas, 226 p.

Cohn, P. 2012. Mitos sobre la caza. In Rodríguez, J. (ed.). Animales no humanos entre animales humanos. Madrid, España DILEMATA. 393p.

de Sousa Santos, B. 2011. Epistemologías del Sur. Utopía y Praxis Latinoamericana. Revista Internacional de Filosofía Iberoamericana y Teoría Social 16(54):17-39.

Del Valle, JI. 1997. La silvicultura desde sus orígenes hasta el siglo XIX. Revista de la Facultad Nacional de Agronomía 50(1):103-130.

Díaz, J; Arana, M; Torres, J; Núñez, SP. 2016. Historia ambiental del Perú. Siglos XVIII y XIX. Lima, Perú, Ministerio del Ambiente, 464 p.

Dourojeanni, MJ. 2009. Crónica Forestal del Perú. Lima, Perú, Ed. San Marcos, Universidad Nacional Agraria La Molina, 727 p.

Dourojeanni, MJ. 2015. Medio siglo de forestería profesional en el Perú. Xilema 28:5-17.
Dourojeanni, MJ. 2019. Esbozo de una nueva política forestal peruana. Revista Forestal del Perú 34 (1):4-20.

Esteve, MA. 2015. Evolución de los paradigmas en conservación de la naturaleza desde una perspectiva ambiental. Revista Eubacteria. Cien años de avances en ciencias de la vida 34:44-50.

FAO (Organización de las Naciones Unidas para la Alimentación y la Agricultura). 2018. El estado de los bosques del mundo - Las vías forestales hacia el desarrollo sostenible. Roma, Italia. FAO, 153 p.

Fuente, M; Ruiz, M; Aquino, C. 2011. El conocimiento indígena contemporáneo. Contribuciones epistémicas para la construcción de la sustentabilidad. In Conde, A; Ortiz, P; Delgado, A; Gómez, F; Granados, L. (Coordinadores). Primer Congreso Nacional Naturaleza y Sociedad. Memoria del Congreso. Tlaxcala, México. p.104-118.

García, R. 2006. Epistemología y teoría del conocimiento. Salud Colectiva 2(2):113-122.

García, L. 2008. Aproximación epistemológica al concepto de ciencia: una propuesta básica a partir de Kuhn, Popper, Lakatos y Feyerabend. Andamios 4(8):185-202.

Gonzáles, I; Gil, L. 2014. ¿Selvicultura o silvicultura? Historia de dos palabras. Revista Montes 118:22-26.

Guariguata, M; Evans, K. 2010. Mejoramiento de la educación forestal universitaria. ¿Debe prestarse más atención a los productos forestales no maderables? Recursos Naturales y Ambiente 59-60:108-116.

Gudynas, E. 2002. Ecología Economía y Ética. El desarrollo sostenible en América Latina. San José, Costa Rica, Universidad Estatal a distancia. 303 p.

Hawley, R; Smith, DM. 1972. Silvicultura práctica. Traducción del Inglés por Jaime. Terradas. Barcelona, España, Ediciones Omega S.A., 544 p Jaramillo, L. 2003. ¿Qué es Epistemología? Mi mirar epistemológico y el progreso de la ciencia. Cinta de Moebio 18:7 p. 
Jones, NA; Shaw, S; Ross, H; Witt, K; Pinner, B. 2016. The study of human values in understanding and managing socialecological systems. Ecology and Society 21(1):15.

Jordan, C.BK; Herz, C; Añazco, M; Miguel, A. 1999. Construyendo cambios. Desarrollo Forestal Comunitario. Una propuesta de manejo participativo de los recursos naturales renovables para el nuevo milenio. Quito, Ecuador, FAO. 446 p.

Kimmins JP. 1997. Balancing Act, Environmental issues in forestry. 2nd ed., Vancouver, Canadá, UBC Press. 305 p.

Klier, G; Casadelrrey, C; Busan, T; di Pasquo, F. 2017. Conservación de la biodiversidad y sus vínculos utilitaristas: cercanías y distancias con Peter Singer y Gifford Pinchot. Revista Metropolitana de Sustentabilidade 7(3): 63-82.

Kwiatkowska, T. 2012. Aldo Leopold y la Ética de la Tierra. Euphyía VI (11): 47-64.

López, N. 2016. Rediseño curricular de la ingeniería forestal en la Universidad nacional de Loja. Loja, Ecuador, Universidad Nacional de Loja. 7p.

Maldonado, C. 2015. Pensar la complejidad, pensar como síntesis. Cinta moebio (54):313324.

Malleux, J. 2013. Cincuenta años de educación forestal en el Perú y América Latina, sus perspectivas a futuro. Xilema 26:10-17.

Malleux, J. 2014. Informe sobre el Taller Regional de Educación Forestal Latinoamericano. - Lima, Perú, Facultad de Ciencias Forestales de la Universidad Nacional Agraria La Molina, 44 p.

Mancuso S; Viola, A. 2015. Sensibilidad e inteligencia en el mundo vegetal. Barcelona, España, Galaxia Gutenberg. 144 p.

Marczak, J; Engelke, P; Bohl, D; Saldarriaga, A. 2016. América Latina y el Caribe 2030: Escenarios futuros. Washington, Estados Unidos, BID \& Atlantic Council. 156 p.

Martínez, A; Ríos, F. 2006. Los Conceptos de Conocimiento, Epistemología y Paradigma, como Base Diferencial en la Orientación Meto- dológica del Trabajo de Grado. Cinta de Moebio $25,5 p$.

Moreno, JA. 2014. Identificación de gérmenes de futuro para la gestión prospectiva del sector forestal colombiano. Bogotá, Colombia, Universidad Nacional de Colombia Facultad Ciencias Económicas, Escuela de Administración y Contaduría Pública. 107 p.

Pérez, M. 2016. La tierra está en nuestras manos. Leonardo Boff. Cuadernos de Teología $\mathrm{X}(1): 165-168$.

Peter, H. 2000. El rendimiento sostenido en el manejo de los bosques: el desafío para Chile de una nueva definición. Bosque Nativo 24:7-10.

Piña, J. 2017. Ciencia ciudadana como emprendimiento de la ciencia abierta: el riesgo del espectáculo de la producción y el acceso al dato. Hacia otra ciencia ciudadana. Liinc em Revista 13(1):47-58.

Prieto, A. 2008. Ordenación de montes y gestión forestal: evolución y nuevas tendencias. Foresta 40:32-45.

Rincón, D. 2018. Los animales como seres sintientes en el marco del principio alterum non laedere: algunos criterios interpretativos. Inciso 20 (1):57-69.

Rodríguez, JM. 2003. Historia forestal y desarrollo forestal sostenible. Cuadernos de la Sociedad Española de Ciencias Forestales 16:297301.

Rodríguez Zoya, LG. 2016. Introducción general a la obra. En: Rodríguez Zoya, L.G. La emergencia de los enfoques de la complejidad en América Latina: desafíos, contribuciones y compromisos para abordar los problemas complejos del siglo XXI. v.1. Buenos Aires, Argentina, Comunidad Editora Latinoamericana. $370 \mathrm{p}$.

Rojo, GE; Martínez R; Rodríguez S; Yuriria. A. 2013. Filosofía de las ciencias forestales. RA XIMHAI 9 (4): 269-272.

Sabogal, A. 2019. El paisaje de los bosques desde una mirada de vuelo de pájaro: Cambio de uso del suelo de los bosques en el Perú. In 
Sabogal, A. (ed.). 250 años de Alexander von Humboldt. Discusiones interdisciplinarias sobre los bosques. Lima, Perú, PUCP. p.69-100.

Salgado, B. (ed). 2015. La ecología funcional como aproximación al estudio, manejo y conservación de la biodiversidad: protocolos y aplicaciones. Bogotá, Colombia, Instituto de Investigación de Recursos Biológicos Alexander von Humboldt. 236 p.

Sanz, G. 2003. Naturaleza y ciencias forestales en Alemania. Una aproximación a la historia de una tradición académica. Cuadernos de la Sociedad Española de Ciencias Forestales 16: 161-166.

Sheil, D; Meijaard, E. 2005. La vida después de la explotación forestal. OIMT Actualidad Forestal Tropical 13(2):12-15.

Shirley, HL. 1964. La enseñanza forestal profesional. UNASYLA No. 75. 18(4):s.p.

Shiva, V. 1991. Abrazar la vida. Mujer, ecología y supervivencia. Montevideo, Uruguay, Instituto del Tercer Mundo. 320 p.

Siebert, H. 1993. Silvicultura cercana a lo natural. En Charlas y Conferencias No. 8. Valdivia, Chile, Universidad Austral de Chile, Facultad de Ciencias Forestales. p:7-10.

UNCCD (Secretaría de la Convención de las Naciones Unidas de Lucha contra la Desertificación). 2017. Perspectiva global de la Tierra. Primera edición. Bonn, Alemania, UNCCD. $238 \mathrm{p}$.

UNIA (Universidad Nacional Intercultural de la Amazonía). 2011. Modificación Planes de Estudio. Carrera de Ingeniería Agroforestal Acuícola de la Facultad de Ingeniería y Ciencias Ambientales. Pucallpa, Perú, UNIA. 137 p.

Velasco, A; Velázquez, D. 2019. El contexto geopolítico de la explotación forestal en la Península de Yucatán, México. Perspectiva Geográfica 24(1):116-137.

Villalobos, R; Carrera, F; de Camino, R; Pierre, J; Flores, W. 2012. Construcción de cultura forestal para el desarrollo: Tres historias de éxito en Latinoamérica. Ponencia presentada en el International Seminar o Scaling Up Rural
Innovations. 7-9 may 2012, Lima Perú; organizado por IRDC Canadá, Fondo Internacional de Desarrollo Agrícola (FIDA), Instituto de Estudios Peruanos (IEP). 19 p.

Zamudio, JG. 2012. Epistemología y educación. Tlalnepantla, Estado de México, México RED TERCER MILENIO S.C. 224 p.

Zanetti, EA; Gómez, JJ; Mostacedo, SJ; Reyes, O. 2017. Cambio climático y políticas públicas forestales en América Latina Una visión preliminar. Santiago de Chile, Chile, CEPAL. 122 p.

Zapata, L; Peña, L. 1998. La historia del bosque y su explotación en el pasado: evidencia arqueológica y etnográfica. Zainak 17:87-99. 